\title{
Flavour Dependence of Form Factors in Heavy Meson Decays
}

F E Close and A Wambach

LIBRARY, R61

15 JUN 1994

RUTHERFORD APPLETON LABORATORY

May 1994 
DRAL is part of the Engineering and Physical

\section{Sciences Research Council}

The Engineering and Physical Sciences Research Council

does not accept any responsibility for loss or damage arising

from the use of information contained in any of its reports or

in any communicalion about its tests or investigations 


\title{
Flavour Dependence of Form Factors in Heavy Meson Decays
}

\author{
FE Close \\ Rutherford Appleton Laboratory, \\ Chilton Didcot, Oxon OX11 OQX, Great Britain. \\ A Wambach \\ Theoretical Physics, \\ Department of Physics, \\ 1 Keble Road, OXFORD, OX1 3NP, Great Britain. \\ RAL-94-041 \\ OUTP - $9409 \mathrm{P}$
}

April 1994

\begin{abstract}
Recently we have shown that due account of Wigner spin rotations is needed to match the ISGW model consistently onto HQET. We now discuss the flavour dependence of this procedure. We find that for finite mass "heavy" quark the universal function $\xi(y=1) \approx 1$ but the slope is proportional to the energy of the spectator quarks, and ratios of form factors are corrected in a way that QCD sum rules seem to require. Predictions for heavy quarks, such as in $B_{c} \rightarrow \psi\left(\eta_{c}\right)$ and to mixed systems, such as $D \rightarrow K$ and $B-\pi$ show a systematic mass dependence that may be confronted with data.
\end{abstract}



The ISGW model [1] is widely used in describing heavy quark transition form factors at low (zero) recoil $\left(y=v \cdot v^{\prime} \approx 1\right)$. In recent works [2]-[5] we have shown that matching this model (and indeed any quark model) onto Heavy Quark Effective Theory (HQET)[6] requires considerable care even when $M_{Q} \rightarrow \infty$, due to the nontrivial recoil and spin rotation structure for light spectator antiquark systems. When these Wigner rotations are consistently accounted for we found that the model with parameters determined from a fit to heavy-light spectroscopy, describes dynamical transitions not just at zero recoil but also is consistent with the leading behaviour for non zero recoil.

In the present note we extend this analysis, motivated by the decay $B_{c} \rightarrow J / \Psi\left(\eta_{c}\right) e \nu$ where both active and spectator quarks are relatively heavy. Although $M_{b} \rightarrow \infty$ is still assumed, consistency requires that mass corrections for the $c$-quark are included, both in its role as active participant and, for the $\bar{c}$, as spectator. The explicit derivation of these corrections then provides us with a tool for calculating transition elements for $B \rightarrow D$, $D \rightarrow K$ and even $B \rightarrow \pi$ in a limited kinematic range.

Our analysis shows that in these cases where HQET fixes the $y=1$ value of a particular form factor to be non-zero, the corrections for finite mass active quarks are small, as is the dependence on the mass of the spectator antiquark. However, for cases where form factors vanish in the $M_{Q} \rightarrow \infty$ zero recoil limit these corrections can be large. Furthermore the slopes show a strong (approximately linear) dependence on the mass of the spectator quark.

We begin by extending ref.[3] to the case of large, but finite, mass active quark, in particular including the Wigner rotation of its spin. With the formalism set up in previous works $[3,4]$ this is achieved immediately. For the general case we consider a meson where the mass of the (active) quark and (spectator) antiquark are different, in this case denoted by $m_{1}$ and $m_{2}$.

In the rest frame the meson wavefunction is written in terms of the quark and antiquark spinors in the form $u(-\vec{k}) \bar{v}(\vec{k})$, where $\vec{k}$ is the relative momentum, and the energy of the first (second) quark is $\omega_{1(2)}=\sqrt{m_{1(2)}^{2}+\vec{k}^{2}}$. This then yields:

$$
M(v=(1, \overrightarrow{0}))=\left(m_{1}+\not K\right)\left(\begin{array}{cc}
0 & X \\
0 & 0
\end{array}\right)\left(m_{2}-\not k\right)\left[4 m_{1}\left(m_{1}+\omega_{1}\right) m_{2}\left(m_{2}+\omega_{2}\right)\right]^{-\frac{1}{2}}
$$

where $K_{\mu}$ denotes the four momentum of quark 1 , and $X$ is either -1 for the pseudoscalar or $\vec{\sigma} \cdot \vec{\epsilon}$ for the vector meson. The equation can be simplified if the internal momenta $K$ and $k$ are decomposed into the direction of $v$ and orthogonal to it, i.e. $K_{\mu}=K_{l} * v_{\mu}+K_{t \mu}$ where $K_{l}=K \cdot v($ and analogously for $k$ ). 
Next the wavefunction is boosted to an arbitrary velocity $v$, where, since $K_{t}=-k_{t}$, we obtain for the pseudoscalar meson:

$$
M(v)=\left(m_{1}+K_{l}-\not \not_{t}\right) \frac{1+\not p}{2} \gamma_{5}\left(m_{2}+k_{l}-\not \not_{t}\right)\left[4 m_{2} m_{1}\left(m_{1}+K_{l}\right)\left(m_{2}+k_{l}\right)\right]^{-\frac{1}{2}}
$$

(for the vector meson, $\notin$ replaces $\gamma_{5}$ ).

In the limit where the spectator (anti)quark does not change its momentum, the matrix element for the pseudoscalar to pseudoscalar transition takes the following form:

$$
\begin{aligned}
& \left\langle P\left(v^{\prime}\right)\left|V_{\mu}\right| P(v)\right\rangle=\xi_{1}(y)\left(v+v^{\prime}\right)_{\mu}+\xi_{2}(y)\left(v-v^{\prime}\right)_{\mu} \\
& =\int \mathrm{d}^{3} k_{t} \mathrm{~d}^{3} k_{t}^{\prime} \delta\left(k_{t}-k_{t}^{\prime}\right) \operatorname{Tr}\left[\left(m_{2}+k_{l}^{\prime}-\not k_{t}^{\prime}\right) \gamma_{5} \frac{\left(1+v^{\prime}\right)}{2}\left(m_{1}+K_{l}^{\prime}-\not k_{t}^{\prime}\right) \Gamma_{\mu} \frac{(1+y)}{2} \gamma_{5}\right] \phi^{*}\left(k_{t}^{\prime}\right) \phi\left(k_{t}\right)
\end{aligned}
$$

where $\phi\left(k_{t}\right)$ is the momentum distribution whose explicit form was discussed in [3,4]; as in those references we employ gaussian wavefunctions with coupling strengths calculated in $[1,7]$. The exponential term is then multiplied by an additional factor $\sqrt{\frac{k_{l}}{k_{0}}}$ in order to render the calculation frame-independent. The normalization factor (inverse square root factor in eq.(2)) is subsumed within the wavefunction term $\phi^{*}$ (and $\phi$ respectively).

The evaluation of $\xi_{1}$ and $\xi_{2}$ is done in the rest frame of the meson after the decay. The agreement with the result in the rest frame before the decay was explicitly checked. For this calculation it is convenient to use:

$$
k_{t}^{\prime}=\left(k_{t}^{\prime} \cdot v\right) \frac{v-y v^{\prime}}{1-y^{2}}+\kappa
$$

with $y=v \cdot v^{\prime}$, and the integration over $\kappa$ is zero. For example the result for $\xi_{1}$ is to order $(y-1)$ :

$$
\begin{aligned}
\xi_{1}(y)= & \int \mathrm{d}^{3} k^{\prime}\left(\beta_{1} \beta_{2} \pi\right)^{-\frac{3}{2}} e^{\left(-k^{\prime 2} A\right)} \sqrt{\frac{m_{1}+\omega_{1}}{2 m_{1}}} \\
& \times\left(1+2(y-1)\left[\frac{1}{4}-\frac{\omega_{2}}{4\left(m_{2}+\omega_{2}\right)}+\frac{k^{\prime 2}}{24\left(m_{2}+\omega_{2}\right)^{2}}-\frac{k^{\prime 2}}{24 \omega_{2}^{2}}-\frac{3 m^{2}+5 k^{\prime 2}}{6 \beta_{1}^{2}}+\frac{m_{2}^{2} k^{\prime 2}+k^{\prime} 4}{6 \beta_{1}^{4}}\right]\right)
\end{aligned}
$$

where $A^{-1}=2\left(\beta_{1}^{2}+\beta_{2}^{2}\right)$. A similar although more laborious calculation provides the form factors for the pseudoscalar to vector meson transition:

$$
\begin{array}{lcc}
\left\langle V\left(v^{\prime}, \epsilon\right)\left|A_{\mu}\right| P(v)\right\rangle & =\rho_{1}(y) \epsilon_{\mu}^{*}+\rho_{2}(y)\left(\epsilon^{*} \cdot v\right) v_{\mu}+\rho_{3}(y)\left(\epsilon^{*} \cdot v\right) v_{\mu}^{\prime} \\
\left\langle V\left(v^{\prime}, \epsilon\right)\left|V_{\mu}\right| P(v)\right\rangle & = & i \eta(y) \epsilon_{\mu \alpha \beta \gamma} v^{\alpha} \epsilon^{* \beta} v^{\prime \gamma}
\end{array}
$$

HQET determines the value of these form factors in the $m_{1} \rightarrow \infty$ limit, namely: $\xi_{1}=\rho_{1} /(y+1)=-\rho_{3}=\eta=\xi$ and $\xi_{2}=\rho_{2}=0$, where $\xi$ is the Isgur-Wise function which is normalized to 1 at $y=1$, the zero-recoil point. 
Luke's theorem [8] states that at this zero-recoil point $\xi_{1}$ and $\rho_{1}$ receive corrections only to order $1 / M^{2}$. From the structure of the expression in (5) it is apparent that the corrections in the mass of the active quark $\left(m_{1}\right)$ are indeed to order $1 / m_{1}^{2}$ for $\xi_{1}(y=1)$. The same result applies for $\rho_{1}$. The other form factors are all corrected by $1 / m_{1}$ terms.

The origin of the absence of $1 / M$ corrections for only two form factors in the quark model description of meson transitions is the following. The current employed during the transition has the form $\bar{u} \Gamma_{\mu} u ; \Gamma_{\mu}$ is diagonal for $\gamma_{0}$ and $\vec{\gamma} \gamma_{5}$ and it is only in these two cases that the upper and lower components of $u$ and $\bar{u}$ do not get mixed, consequently $1 / M$ terms do not appear. In the zero-recoil frame the expressions for these currents are:

$$
\begin{aligned}
\left\langle P(v)\left|\Gamma_{0}\right| P(v)\right\rangle & =2 v_{0} \xi_{1}(y=1) \\
\langle V(v, \epsilon)|\vec{A}| P(v)\rangle & =\rho_{1}(y=1) \vec{\epsilon}^{*}
\end{aligned}
$$

Therefore $\xi_{1}$ and $\rho_{1}$ are protected for corrections in $1 / m_{1}$.

Let us now consider mass effects for the nonperturbative corrections. In the ISGW model of present interest, these are subsumed in a phenomenologically inspired non-relativistic Coulomb plus linear potential [1] and variational solutions to the Schrödinger problem were then found based on harmonic oscillator wavefunctions. The parameter in this gaussian wavefunction which was optimized by a fit to spectroscopy is the oscillator strength $\beta \equiv \sqrt{m \Delta E}$ for particle mass $m$ with excitation energy $\Delta E$. Empirically $\Delta E$ is approximately flavour independent [9]. In Table 1 we display the oscillator strengths for various flavour systems, as calculated in $[1,7]$, which confirms the approximate flavour independence of $\Delta E$ when the reduced mass $m=m_{1} m_{2} /\left(m_{1}+m_{2}\right)$ is used.

\begin{tabular}{|c||c|c|c|c|c|c|c|}
\hline meson & $\bar{B}_{c}$ & $B_{s}$ & $B$ & $J / \Psi$ & $D$ & $K$ & $\pi$ \\
\hline$\beta(\mathrm{GeV})$ & 0.82 & 0.51 & 0.41 & 0.66 & 0.39 & 0.34 & 0.31 \\
\hline$\Delta E_{1}(\mathrm{GeV})$ & 0.40 & 0.47 & 0.51 & 0.26 & 0.46 & 0.35 & 0.29 \\
\hline$\Delta E_{2}(\mathrm{GeV})$ & 0.53 & 0.52 & 0.54 & 0.51 & 0.55 & 0.56 & 0.58 \\
\hline
\end{tabular}

Table 1: In row 2 different coupling strengths for different mesons as given in [1, 7] are displayed. In row $3 \Delta E_{1}=\beta^{2} / m$ is determined where $m$ is the mass of the light constituent (anti)quark. $\Delta E_{2}$ is calculated with $m$ being the reduced mass of the system. Quark masses employed are the constituent masses: $m_{u}=0.33 \mathrm{GeV}, m_{s}=0.55 \mathrm{GeV}, m_{c}=1.7 \mathrm{GeV}$ and $m_{b}=5.12 \mathrm{GeV}$. Note the stability of $\Delta E_{2}$.

In the $M_{Q} \rightarrow \infty$ limit, the reduced mass $m$ becomes that of the light (anti) quark. However, for a consistent description of $1 / M$ effects, the dependence of $\beta$ on the heavy quark mass has to be taken into account. In particular, with 
$\beta=\sqrt{m_{1} m_{2} \Delta E /\left(m_{1}+m_{2}\right)} \approx \sqrt{m_{2} \Delta E}\left(1-m_{2} /\left(2 m_{1}\right)\right)$, effects in $1 / M$ are entering the scene. However, in the zero recoil limit the form factors $\xi_{1}$ and $\rho_{1}$ are still protected against picking up nonperturbative $1 / M$ corrections, due to the normalization of the Isgur-Wise function. In the general case, where the wavefunction for meson $1(2)$ gets corrected by $1 / M_{1(2)}$ terms, the overall correction to a form factor has the structure:

$$
\Delta_{\xi}=\int \mathrm{d}^{3} k\left[\left.\Phi^{*}\left(k, \beta_{2}\right) \frac{d \Phi\left(k, \beta_{1}\right)}{d \beta_{1}}\right|_{\beta_{1}=\beta_{2}} \Delta\left(\beta_{1}\right)+\left.\frac{d \Phi^{*}\left(k, \beta_{2}\right)}{d \beta_{2}}\right|_{\beta_{1}=\beta_{2}} \Phi\left(k, \beta_{1}\right) \Delta\left(\beta_{2}\right)\right]
$$

However, this form factor is normalized at zero recoil $[10,11]$ in the infinite mass limit where $\beta_{1}=\beta_{2}=\beta$ :

$$
\int \mathrm{d}^{3} k \Phi^{*}(k, \beta) \Phi(k, \beta)=1
$$

This result is independent of $\beta$ and therefore

$$
\int \mathrm{d}^{3} k \Phi^{*}(k, \beta) \frac{d \Phi(k, \beta)}{d \beta}=0
$$

so that $\Delta_{\xi}=0$ in eq.(8). A similar conclusion has been made in ref.[12].

For the following numerical results we performed the calculation in the limit where the active heavy quark before the decay is infinitely heavy (in general the bottom quark), however after the decay it picks up mass corrections (usually the charm quark). In table 2 the results for a particular case, namely $B \rightarrow D^{(*)}$ are displayed (i) in the HQET limit and (ii) with mass corrections for the charm quark in the $D$ meson. To see the dependence of the numerical values on the mass of the spectator quark we display in row 4 the same form factors for the decay $B_{s} \rightarrow D_{s}^{(*)}$.

\begin{tabular}{|c||c|c|c|c|c|c|}
\hline form factor & $\xi_{1}$ & $-\xi_{2}$ & $\rho_{1}$ & $\rho_{2}$ & $-\rho_{3}$ & $\eta$ \\
\hline HQET-limit & $1.00-1.40 \epsilon$ & 0 & $2.00-1.81 \epsilon$ & 0 & $1.00-1.40 \epsilon$ & $1.00-1.40 \epsilon$ \\
\hline finite $m_{c}$ & $1.01-1.43 \epsilon$ & $0.10-0.12 \epsilon$ & $2.01-1.69 \epsilon$ & $0.17-0.32 \epsilon$ & $0.98-1.30 \epsilon$ & $1.16-1.62 \epsilon$ \\
\hline$B_{s} \rightarrow D_{s}^{(*)}$ & $1.00-1.64 \epsilon$ & $0.14-0.18 \epsilon$ & $2.00-2.08 \epsilon$ & $0.22-0.42 \epsilon$ & $0.98-1.48 \epsilon$ & $1.19-1.91 \epsilon$ \\
\hline
\end{tabular}

Table 2: In this table the numerical results for the different form factors for $B \rightarrow D^{(*)}$, defined in eq. $(6)$, to order $(y-1)$ are displayed $(\epsilon \equiv(y-1))$. Row 2 shows the results in the HQET limit. Row 3 includes $1 / m_{c}$ corrections and in row 4 the spectator antiquark is the strange antiquark instead of the up or down.

In general the data behave in the following way: If HQET predicts a nonzero value for a form factor at a special point, i.e. the zero-recoil point, the modifications induced by mass effects are not significant. Furthermore, a variation of the spectator quark mass changes this result only slightly. However, values which are not restricted by HQET (such as the slope of 
the form factor, coefficient of $\epsilon$ ) do show a very strong dependence on the spectator quark mass; for example, the slope of $\xi_{1}$ turns out to be nearly proportional to the mass of the spectator quark (see figure 1). This finds its cause in the non-relativistic prediction, where $\rho^{2}=\frac{m^{2}}{2 \beta^{2}}=\frac{m}{2 \Delta E}$, together with the empirical flavour independence of $\Delta E$. Similar results have been noted by Voloshin [13] in a different context. Deviations to this linear dependence on the spectator quark mass are induced by relativistic effects.

In figure 2 we plot the form factor $\xi_{1}$ as a function of $(y-1)$ for different meson transitions in a limited range where $\xi_{1}$ exhibits a linear dependence in $(y-1)$. Note that the slopes for $B \rightarrow \pi, D \rightarrow K$ and $B r r o w D$ are approximately parallel, reflecting their common light spectator (anti)quark; $D_{s} \rightarrow \bar{s} s$ is analogously parallel to $B_{s} \rightarrow D$, though with a larger slope due to the heavier strange quark mass and $B_{c} \rightarrow a_{c}$ has the steepest slope due to the massive charmed spectator.

Comparison with experiments are shown in figure 3.

Effects induced by finite mass corrections are usually discussed in the context of the functions $R_{1}$ and $R_{2}$ which were proposed by Neubert [14] as a measure of symmetry breaking effects. They read (in our parametrization of the form factors):

$$
\begin{aligned}
& R_{1}(y)=2 \frac{\eta(y)}{\rho_{1}(y)}\left(1+\frac{1}{2}(y-1)\right) \\
& R_{2}(y)=-2 \frac{\rho_{2}(y)+r \rho_{3}(y)}{\rho_{1}(y)}\left(1+\frac{1}{2}(y-1)\right)
\end{aligned}
$$

where $r=M_{D^{*}} / M_{B}$. In the HQET limit $R_{1}=R_{2}=1$, however finite mass corrections as well as QCD correction terms modify this result.

In the ISGW model, the ratios $\eta(y) / \rho_{1}(y)$ and $\left(\rho_{2}(y)+r \rho_{3}(y)\right) / \rho_{1}(y)$ are constant, so that the $y$ dependences of $R_{1}$ and $R_{2}$ are that of the common factor $\left(1+\frac{1}{2}(y-1)\right)$. Explicit evaluation of the ratios leads to:

$$
\begin{aligned}
& R_{1}(y)=1.01+0.50(y-1)+\mathrm{O}\left((y-1)^{2}\right) \\
& R_{2}(y)=0.91+0.45(y-1)+\mathrm{O}\left((y-1)^{2}\right)
\end{aligned}
$$

In our computation the velocity dependence is more subtle. We find

$$
\begin{aligned}
& R_{1}(y)=1.15-0.07(y-1)+\mathrm{O}\left((y-1)^{2}\right) \\
& R_{2}(y)=0.91+0.04(y-1)+\mathrm{O}\left((y-1)^{2}\right)
\end{aligned}
$$

which in sign as well as in magnitude approaches the estimate based on QCD sum rules, given in [6]:

$$
\begin{aligned}
& R_{1}(y)=1.35-0.22(y-1)+\mathrm{O}\left((y-1)^{2}\right) \\
& R_{2}(y)=0.79+0.15(y-1)+\mathrm{O}\left((y-1)^{2}\right)
\end{aligned}
$$


The remaining small discrepancy can be partially explained by noting that $R_{1}$ receives substantial short-distance corrections proportional to $\alpha_{s}\left(m_{c}\right)$ which are not included in the quark model.

We have shown so far the application of the consistent model for space like form factors, where it appears be a success. There is much concern about the flavour (mass) dependence of timelike form factors, namely the decay constant of heavy mesons. HQET predicts that the decay constant has to scale like $M^{-1 / 2}$. In particular this implies: $f_{B} \sqrt{M_{B}}=f_{D} \sqrt{M_{D}}$.

However, lattice calculations [15] determine $f_{B}=180 \pm 40 \mathrm{MeV}$ and $f_{D}=200 \pm 30 \mathrm{MeV}$, which differs from the HQET prediction: $f_{D}=\sqrt{\frac{M_{B}}{M_{D}}} f_{B}=300 \pm 70 \mathrm{MeV}$. QCD sum rules imply a similar behaviour [16].

In the quark model formalism of this paper, the decay constant is to be calculated via:

$$
\begin{aligned}
& \left\langle 0\left|A_{\mu}\right| P(v)\right\rangle \propto \int \operatorname{Tr}\left[\left(m_{1}+K_{t}\right) \frac{(1+y)}{2} \gamma_{5}\left(m_{2}-\not k\right) \gamma_{\mu} \gamma_{5}\right]\left[4 m_{2} m_{1}\left(m_{1}+K_{l}\right)\left(m_{2}+k_{l}\right)\right]^{-\frac{1}{2}} \phi\left(k_{t}\right) \\
& \propto \quad v_{\mu} \int\left[\left(m_{1}+\omega_{1}\right)\left(m_{2}+\omega_{2}\right)+k_{t}^{2}\right]\left[4 m_{2} m_{1}\left(m_{1}+K_{l}\right)\left(m_{2}+k_{l}\right)\right]^{-\frac{1}{2}} \phi\left(k_{t}\right) \\
& \propto \quad v_{\mu} \int\left[\sqrt{1+\frac{\omega_{2}}{m_{2}}}-\frac{1}{m_{1}}\left(\sqrt{\frac{\omega_{2}}{m_{2}}-1} * \frac{|k|}{2}\right)\right] \phi\left(k_{t}\right)+0\left(\frac{1}{m_{1}^{2}}\right)
\end{aligned}
$$

The Wigner-rotation of the spin of the heavy quark thus does correct the HQET expression, decreasing the value of the decay constant and thereby bringing $f_{B}$ and $f_{D}$ closer to each other. The numerical importance of the $1 / m_{1}$ correction term is in this model $3 \%$ for the $\mathrm{B}$-meson and $15 \%$ for the D-meson. This effect alone, however, is too small to explain the large deviation from the HQET limit as calculated in lattices and QCD sum rules (see above). So a description of the (timelike) decay systematics remains elusive in this approach, whereas the spacelike shows agreement with available data. Data on $B_{c} \rightarrow \psi\left(\eta_{c}\right)$ at one extreme and $B \rightarrow \pi$ at the other will show the range of applicability of this model in the spacelike domain. If it turns out to be successful, then the problems in the timelike region will be highl ighted.

This feature in the timelike region may be due to the specific probing of the wavefunction at one point $(r \rightarrow 0)$, in contrast to spacelike form factors which "average" over the whole wavefunctions. But in any case it is intersting to note that the corrections induced by the Wigner rotation of the spin of the heavy quark do correct the decay constant in the right direction, which suggests that relativistic effects may dominate the decay amplitude and therefore lie beyond the spectroscopic-based non relativistic models. 


\section{References}

[1] N. Isgur, D. Scora, B. Grinstein, M. Wise, Phys. Rev. D39 (1989) 799

[2] F. E. Close and Z. Li, Phys. Lett. B237 (1992) 143

[3] F. E. Close and A. Wambach, Nucl. Phys. B412 (1994) 167

[4] A. Wambach, OUTP 93-28P, hep-ph 931222

[5] A. Wambach, OUTP 94-01P, hep-ph 9403207, to appear in Z. Phys. C

[6] For a review see: M. Neubert, Heavy Quark Symmetry, SLAC-PUB-6263, hep-ph 9306320, to be published in Physics Reports

[7] M. Lusignoli and M. Masetti, Z. Phys. C51 (1991) 549

[8] M. E. Luke, Phys. Lett. B252 (1990) 447

[9] Review of Particle Properties, Phys. Rev. D45, Part 2, (June 1992)

[10] F. E. Close, G. J. Gounaris, Phys. Lett. B149, (1984) 209

[11] N. Isgur and M. Wise, Phys. Lett. B 232 (1989) 113

N. Isgur and M.B. Wise, Phys. Lett. B 237 (1990) 527

[12] J. F. Amundson, Phys. Rev. D 49 (1994) 455

[13] M. B. Voloshin, Phys. Rev. D 46 (1992) 3062

[14] M. Neubert, Phys. Rev. D46 (1992) 3914

[15] C. T. Sachrajda, Talk given at the International Europhysics Conference in High Energy Physics, Marseille, France, July 1993

[16] C. A. Dominguez, SISSA 139/39/EP, hep-ph 9309260, presented at the 3rd Workshop on the $\tau$ /charm Factory, Marbella, Spain, June 1993

[17] H. Albrecht et al. (ARGUS Collab.), Z. Phys. C57 (1993) 533 


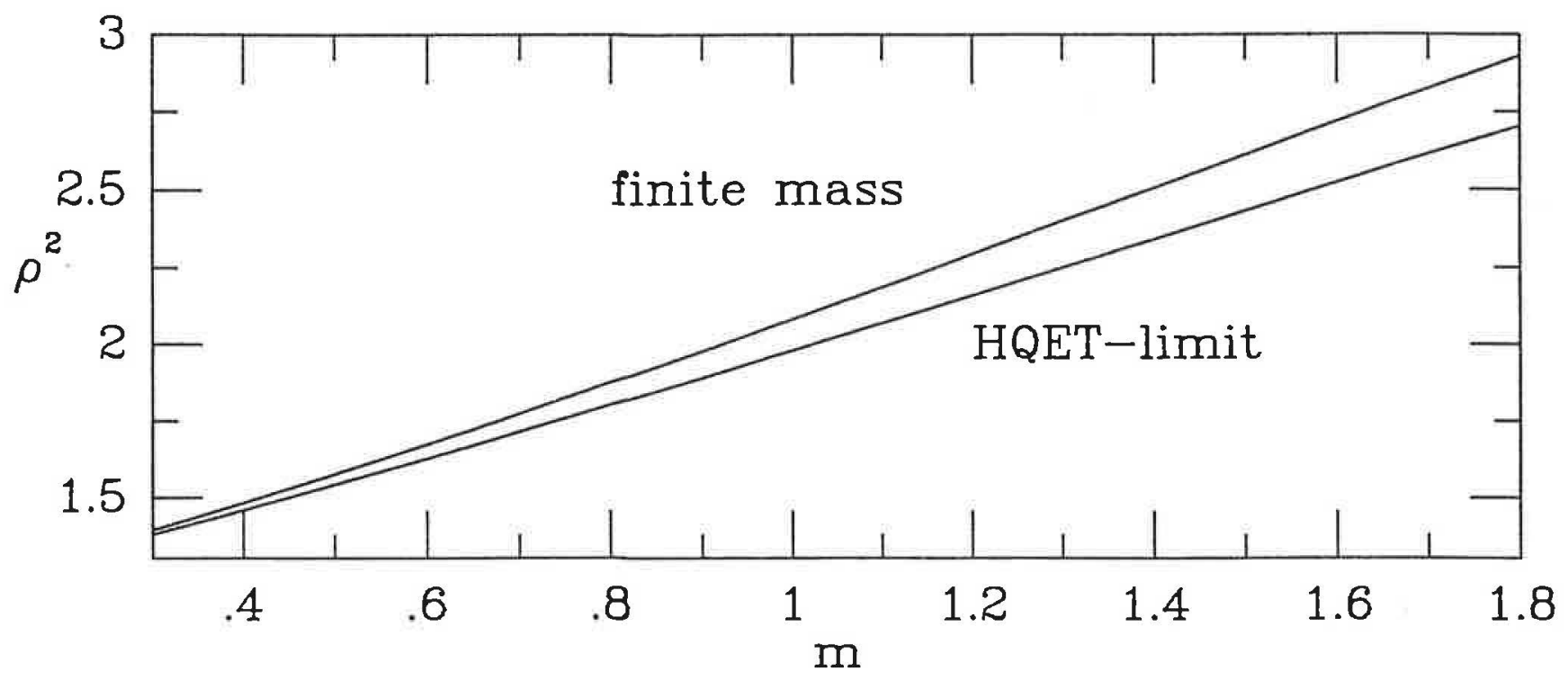

[1] The figure shows the dependence of the charge radius $\rho^{2}$ on the mass of the spectator antiquark $(m)$. (i) in the HQET-limit and (ii) with a finite charm quark mass, $\left(m_{c}=1 . \bar{f}\right.$ GeV).

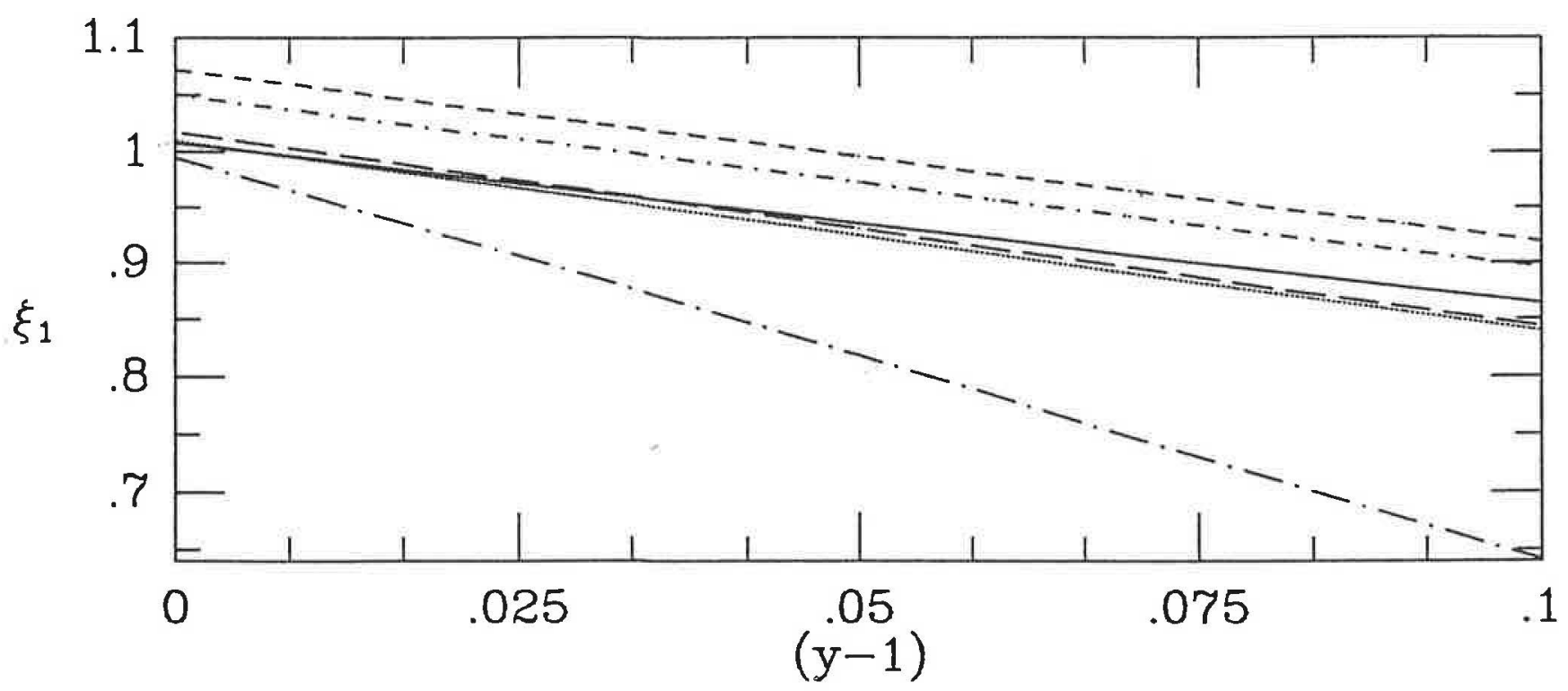

[2] The form factor $\xi_{1}$ is plotted for different meson transitions. From top to bottom they are: $B \rightarrow \pi$ (short dash). $D \rightarrow K$ (dot - short dash), $D_{s} \rightarrow \vec{s} s$ (long dash), $B_{s} \rightarrow D_{s}$ (dotted), $B-D$ (solid), $B_{c}-\eta_{c}$ (dot - long dash) 


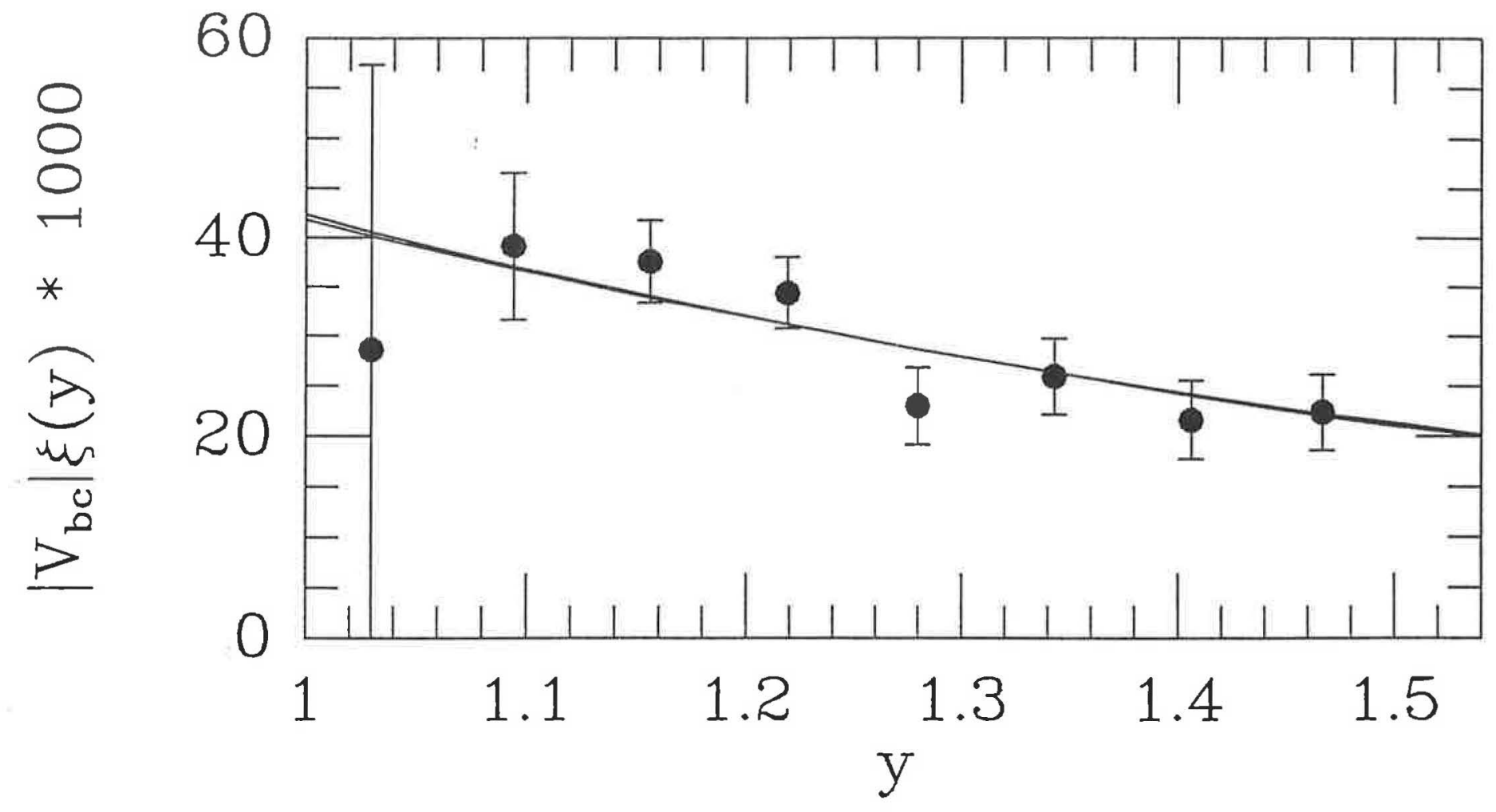

[3] Comparism of the model with experimental data from ARGUS [16] for $\left|V_{b c}\right|$ in the infinite mass limit and with a finite charm quark mass. Note that the mass dependence is nugatory. 


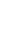



